\title{
A New Geometric Calibration Algorithm for Multi-projector Display System
}

\author{
Chao Xiao ${ }^{1, a, *}$, Kaimin Chen ${ }^{2, b}$ \\ ${ }^{1}$ College of Optoelectronic Technology, Chengdu University of Information Technology, Chengdu, \\ China \\ ${ }^{2}$ College of Computer Science, Sichuan University, Chengdu, China \\ axiaochao@cuit.edu.cn, ${ }^{\mathrm{a}}$ chenkaimin@scu.edu.cn \\ *corresponding author
}

Keywords: Multi-projector display, Geometric calibration, Structured light projection

\begin{abstract}
Aiming at the problem of geometric warp in multi-projector display system, a new auto-geometric calibration algorithm based on structured light projection technique was proposed. First, the geometric mapping relation among display wall, projector and camera was defined. And then camera was used to capture longitude and latitude spot-array and structured fringes on display wall. The longitude-latitude value in global coordinate for each pixel on projector frame buffer was calculated by the feature extraction of images from the camera. The above process implemented the non-linear geometric calibration of projected images. The calibration of camera and prior knowledge of analytic expressions for display wall were not necessary for the proposed algorithm. Furthermore, it was suitable for any smooth display walls. Both computer simulation experiments and real applications on virtual airport system showed that the proposed method could provide higher calibration accuracy effectively.
\end{abstract}

\section{Introduction}

With the increasingly wide influence of virtual reality technology in the fields of business, entertainment and scientific research, the demand of the market for large screen display with highresolution has increased and multi-projector display technique has attracted more and more attention. Furthermore, a lot of excellent systems have emerged ${ }^{[1-2]}$. Geometric calibration, brightness calibration and edge blending are the key technologies of multi-projection display system, and the establishment of pixel level mapping relation between projector and camera is the premise and basis.

We will obtain deformed images if projecting images onto display wall directly. In order to obtain correct images on display wall, the projector needs project pre-deformed images. Only in this way can we counteract geometric dislocation which is caused by arbitrary placement of projector and irregular display wall. The above processes are geometric calibration. Building precise geometric mapping relation between projector and display wall is the key point of geometric calibration. Ref.3 uses feature lines to build the mapping relation between projector and display 
wall. But the calibration accuracy is low when the light of projector is weak. Ref.4 proposes an image alignment algorithm based on twice transformation. It calibrates the camera and projector in advance, and estimates the parameters of quadratic surface. Ref.5 uses feature bar to substitute for feature line, which increases inspection accuracy and Ref.6 builds mapping relation by using subdivision surface technology to thicken original sparse feature grids. Ref.7 calculates the transformation matrices between display wall and projector by the feature extraction of images from camera.

Based on structured light projection technique, we propose a new geometric calibration algorithm, which uses the phase unchanged relation during light transmitting process to establish the sub pixel corresponding relation between the projector image and camera image. The principle is that the phase value of synonymous phase points between standard fringe diagram and deformed fringe diagram is equal and this relation does not change during light transmitting process ${ }^{[8]}$. This method does not need any prior knowledge about projector, display wall and camera, and simplifies the processes of the existing algorithms. It directly obtains the mapping relation, and improves the accuracy of geometric calibration.

\section{Geometric Calibration of Multi-projection System}

Every feature point in standard geometric calibration image (SGCI) has an exclusive identification number. Because the recognized feature points are stored in a matrix, the identification number is expressed as row index and column index of the matrix.

Each feature point has exclusive longitude-latitude value, so the identification number and longitude-latitude value are one to one correspondence for a feature point. $(c x, c y)$ is set as pixel in the camera image, $(b x, b y)$ is pixel in the frame buffer image, and $(p x, p y, p z)$ is pixel in the projection image. When the projector projects the image onto display wall, there is:

$$
f_{1}: \mathbf{R}^{2} \rightarrow \mathbf{R}^{3}, \quad f_{1}(b x, b y)=(p x, p y, p z)
$$

This process is a nonlinear mapping process. For the same reason, when the camera captures an image from the display wall, there is:

$$
f_{2}: \mathbf{R}^{3} \rightarrow \mathbf{R}^{2}, \quad f_{2}(p x, p y, p z)=(c x, c y)
$$

This process is also a nonlinear mapping process. These two processes are integrated to give the pixel mapping relation from frame buffer image to camera image,

$$
(c x, c y)=f_{2}\left(f_{1}(b x, b y)\right)=g(b x, b y)
$$

Here $g=f_{2} f_{1}$. Fig. 1 shows the twice non-linear mapping processes.

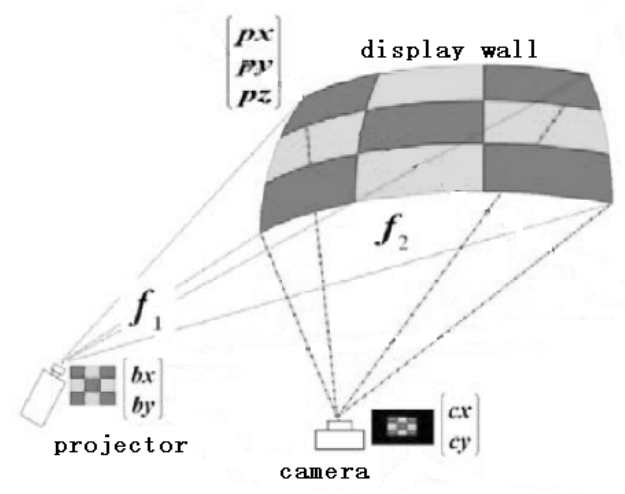

Figure 1 Mapping relation among projector, camera and display wall 
In order to get precise pixel alignment relation between frame buffer image and camera image, we need to know the mapping relation $g$. Since we don't know the analytical expressions of the surface of the display wall, it is difficult to obtain $g$ directly.

\section{Geometric Calibration Based on Structured Light}

As mentioned above, the key of geometric calibration is building the pixel mapping relation between frame buffer image and projection image. In this paper, we establish the mapping relation using structured light projection technique.

\subsection{Principle of the Algorithm}

We set SGCI as the reference criterion. There are many feature spots on SGCI and each spot has an exclusive longitude-latitude value. The purpose of setting the longitude-latitude lattice diagram is to establish the global coordinate.

For a pixel $p$ on frame buffer image, we could find a series of phase-equal points whose phase value are equal to $p$ 's vertical phase value in the camera's vertical phase unwrapping diagram. Using these phase-equal points, we could fit a phase-equal line in vertical direction. And in the similar way, an equal-phase line in horizontal direction could be fitted. The intersection point of the two phase-equal lines is the corresponding pixel (sub pixel) on camera image of pixel $p$ on frame buffer. After finding all the corresponding pixels of frame buffer image pixels, we establish the mapping relation between projector and camera. Fig. 2 shows the mapping diagram in the onedimensional space.

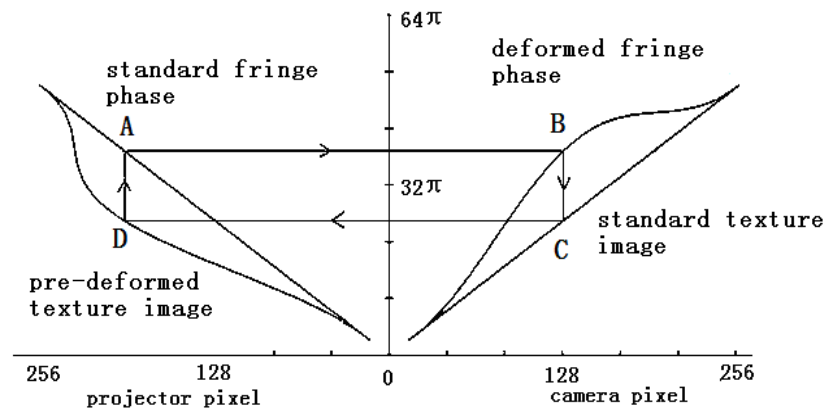

Figure 2 Mapping relation between projector image and camera image

\subsection{Implementation Process of the Algorithm}

The algorithm flow is described as follows:

(1) For each projector, a series of horizontal and vertical sinusoidal fringes are projected respectively, and there is phase shift between adjacent fringes; a chessboard image is projected and its central corner point is used as the starting point for phase unwrapping; project the corresponding pre-deformed longitude-latitude lattice diagram of each projector.

(2) Take pictures of the above sinusoidal fringe images, lattice image and chessboard image. Take note of capturing pictures of the entire characteristic image and keeping the position of the camera fixed;

(3) Carry out the phase unwrapping of the fringe images. Implement the phase unwrapping of the standard sinusoidal fringes and deformed fringes in both horizontal and vertical directions using diamond phase unwrapping algorithm. The unwrapped starting point is determined by the central corner point of the chessboard image; 
(4) Use the gravity method with threshold value to extract feature points in the lattice diagram. Use scattered data ordering algorithm ${ }^{[9]}$ to obtain the identification number of each feature point. So we obtain the longitude-latitude values of each feature point;

(5) Calculate the pixel corresponding relation between the frame buffer image and the camera image. After the phase unwrapping is completed, the establishment of the mapping relation is carried out according to section 3.1;

(6) Use the obtained geometric calibration data to generate pre-deformed images and fusion mask, and then measure the brightness response curve of the projector, which are used for brightness calibration and edge blending.

\section{Experiment Results}

The experimental environment is virtual airport system, which is composed of 24 Panasonic projectors in the way of 10+10+4 from below, as shown in Fig. 3.

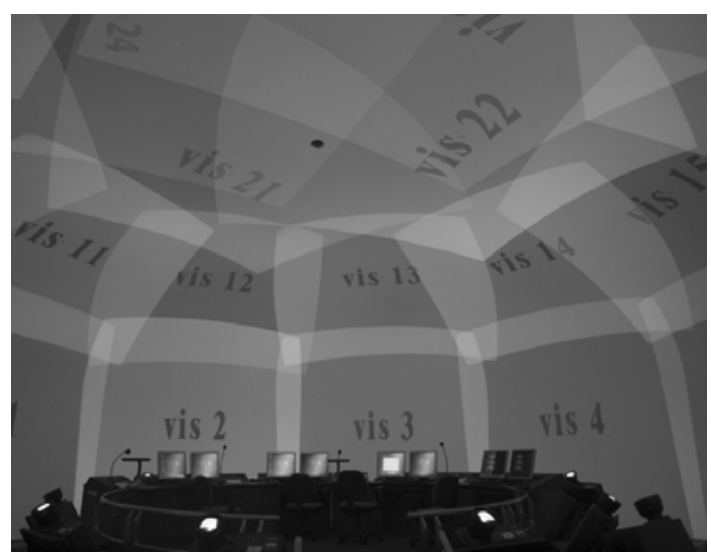

Figure 3 The projection structure of projectors

The display wall is designed as $13 \mathrm{~m}$ in diameter, $6.5 \mathrm{~m}$ in height, the bottom is cylindrical surface, and the top is irregular spherical surface with $13 \mathrm{~m}$ in diameter and $2.7 \mathrm{~m}$ in height. The exact analytical expression of the display wall is not unknown because of construction errors, gravity distortion and other factors. Fig. 4 shows the images that generated in the process of experiment.

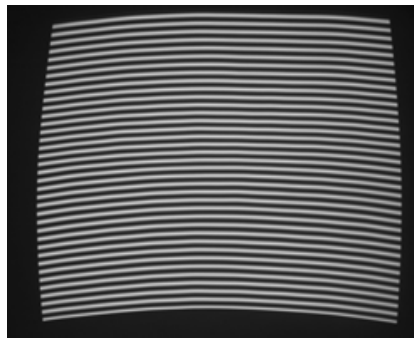

(a)

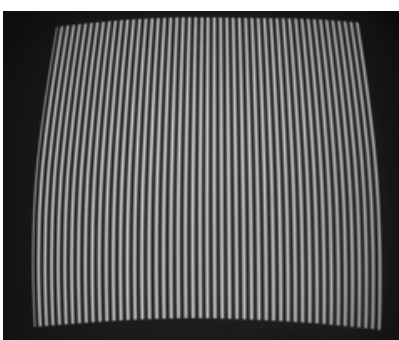

(b)

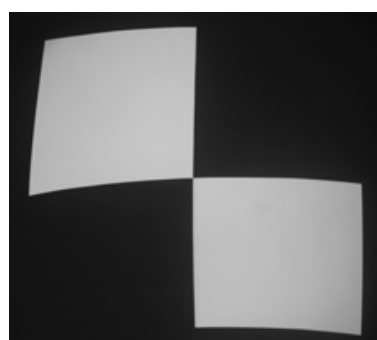

(c)

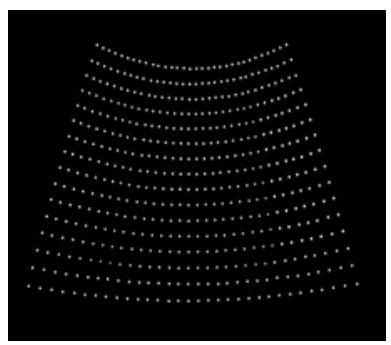

(d)

Figure 4 (a) Horizontal fringe (b) Vertical fringe (c) Checkerboard image (d)Longitude-latitude points

Fig. 4(a) is one of horizontal projecting fringes and Fig. 4(b) is one of vertical projecting fringes captured by camera. Due to the modulation of the display wall, the fringes are deformed. Fig. 4(c) is chessboard image projected for the alignment of unwrapping starting points, and the central corner point of the image is used as the starting point of the phase unwrapping. Fig. 4(d) is camera image with standard longitude-latitude lattice diagram. After the establishment of the mapping relation 
between the projector image and the display wall image through the algorithm proposed in this paper, each frame of texture image in frame buffer is changed into a pre-deformed image using this mapping relation. And then the visual image without deformation could be obtained after projecting pre-deformed image onto the display wall.

The resolution of the visual image is $1400 \times 1050$ and the program is working based on OSG rendering engine. Fig. 5 shows the virtual airport images.

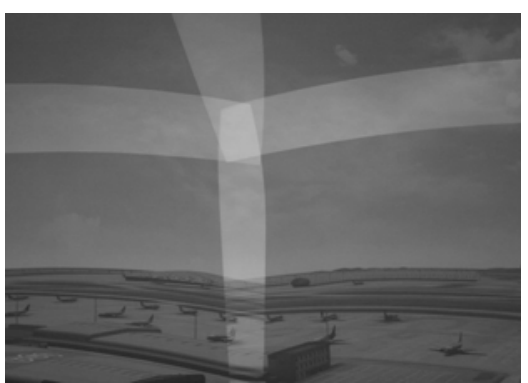

(a)

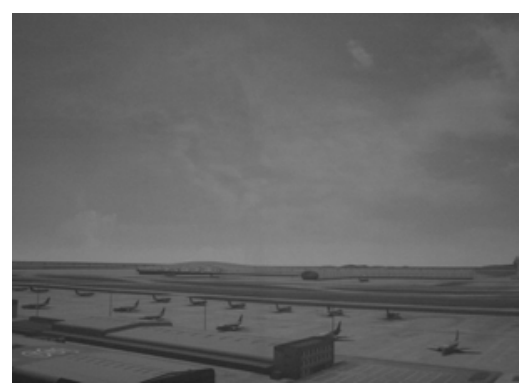

(b)

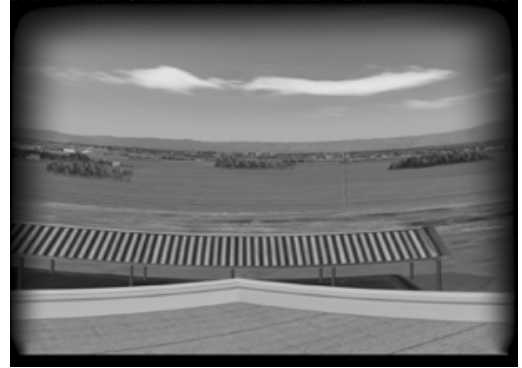

(c)

Figure 5 (a) Image before calibration (b) Image after calibration (c) Image after using fusion mask

Fig. 5(a) is camera image on display wall before geometric calibration. It could be seen that the runway is curved due to the modulation of display wall. Fig. 5(b) shows the image on display wall after geometric calibration, and the runway is straight at this time. Fig. 5(c) is the image obtained after using fusion mask.

\section{Conclusions}

A geometric calibration algorithm based on structured light projection for multi-projection display system is proposed. It is not necessary to know the prior knowledge of display wall and calibrate the camera. The image mapping relation could be easily built by projecting several structured fringes. There are two sets of phase values for arbitrary pixel on the frame buffer of projector. A series of positions in camera coordinate are found which have the same phase value with the pixels on projector. After fitting two phase-equal lines using the phase-equal points, we could build sub-pixel corresponding relation between camera and projector by obtaining the intersection point of the two phase-equal lines. Experimental results show that the proposed method could provide higher calibration accuracy.

\section{Acknowledgements}

This research was financially supported by the Scientific Research Foundation(KYTZ201516) and the Project of Sichuan Provincial Department of Education (17ZB0084).

\section{References}

[1] Raij A, Gill G, Majumder A, et al. "PixelFlex2: a comprehensive, automatic, casually-aligned multi-projector display," Proceeding IEEE International Workshop Projector-Camera System. Washington DC, USA: IEEE Computer Society, pp.1-8, 2003.

[2] X. H. Wang, W. Hua, H. Lin, et al. "Screen calibration techniques for multi-projector tiled display wall," Journal of Software, vol.18, no.11, pp.2955-2964, 2007.

[3] Surati R.J. "Scalable self-calibrating display technology for seamless large-scale displays," Cambridge, Massachusetts: Massachusetts Institute of Technology, 1999. 
[4] Ramesh R, van Jeroen B, Thomas W. "Quadric transfer for immersive curved display," Proceedings of EUROGRAPHICS. Washington DC, USA: IEEE Computer Society, vol.23, no. 3, pp.451-460, 2004.

[5] X. H. Wang, H. B. Yang, H. Lin. "Geometry calibration for multi-projector tiled display wall," Journal of Computer-aided Design \& Computer Graphics, vol.20, no.6, pp.707-712, 2008.

[6] J. Zhang, B. P. Wang, X. F. Li. "Method of image alignment for calibration of multi-projector displays," Journal of Image and Graphics, vol.16, no.2, pp.293-299, 2011.

[7] S. Z. Wang, J. Yang. "Auto-nonlinear geometry calibration and edge blending of multi-projector display system," Journal of Shanghaijiaotong University, vol.42, no.4, pp.574-578, 2008.

[8] C. Xiao, X. Y. Su, H. L. Jing. " A new method for generation of inverse projected fringe," ACTA OPTICA SINICA, vol.28, no.11, 2008.

[9] B. P. Wang. "The research on image fusion key technologies of synthetic vision system," Ph.D. Dissertation, Sichuan University, 2009. 